\title{
Celestial Mechanics in the XXIst century - challenges
}

\author{
Vivian M. Gomes ${ }^{1, a}$, Antônio F.B.A. Prado ${ }^{2}$, Cristiano F. de Melo ${ }^{3}$, \\ Elbert E.N. Macau ${ }^{4}$, and Othon C. Winter ${ }^{1}$ \\ ${ }^{1}$ Unesp, São Paulo State University, Guaratinguetá, SP, Brazil \\ ${ }^{2}$ Instituto Nacional de Pesquisas Espaciais, INPE, São José dos Campos, SP, Brazil \\ ${ }^{3}$ Universidade Federal de Minas Gerais, UFMG, Belo Horizonte, MG, Brazil \\ ${ }^{4}$ Universidade Federal de São Paulo, UNIFESP, São José dos Campos, SP, Brazil
}

Received 17 April 2020

Published online 29 May 2020

\begin{abstract}
This special issue of the European Journal of Physics: Special Topics entitled Celestial Mechanics in the XXIst Century published a set of 15 papers aiming to put in perspective the main problems that are being addressed by the researchers in this area. Our goal is to draw attention to the challenges that they represent so that new approaches may be conceived to foster better and more effective solutions of them. In particular, it is shown points related to a new type of creep tide theory, studies related to the risk of collision of space vehicles with space debris, motion near NEAs, strategies to avoid a collision of an asteroid with the Earth and decay of small satellites in the atmosphere of the Earth are included, since they are hot topics of current research.
\end{abstract}

\section{Introduction}

The Celestial Mechanics is a branch of Astronomy and its goal is to study and understand phenomena involving the motion of bodies under gravitational attraction. Its origin goes back to the time of ancient civilizations, first considering the periodicities in the celestial bodies' motion to build calendars, forecast seasons and astronomical phenomena. Over the last few centuries, the works of many formidable mathematicians, physicists and engineers such as Johannes Kepler (1572-1630), Galileo Galilei (1564-1642), Isaac Newton (1642-1727), Leonhard Euler (1707-1783), Jean le Rond d'Alambert (1717-1783), Giuseppe Lagrange (1736-1813), Adrien-Marie Legendre (1752-1833), Hamilton William (1805-1865), Henri Poincaré (1854-1912), Aleksandr Lyapunov (1857-1918), Walter Hohmann (1880-1945), Giussepe Colombo (19201984) and many others $[1,2]$ as well as the import advent of the high performance computing in the 20th century allowed to develop the modern Celestial Mechanics. Thanks to this modern science and its many areas, especially Astronautics, the space is awash of satellites providing fast communication among people, companies and governments, navigation, transportation of people and the trade of goods, public safety, natural resources exploitation, etc. Probes launched into deep space have brought an extraordinary knowledge on the origin and evolution of our solar system

a e-mail: vivianmartinsgomes@gmail.com 
and we begin to understand better other solar systems through the universe. The modern Celestial Mechanics and Astronautics is playing a relevant role in shaping of the lifestyle since the end of 20th century and its development will continue to be an important tool for mankind evolution. If at some point of the 21st century the humanity is driven to occupy and colonize the Solar System, the Celestial Mechanics and Astronautics will be the bases for this journey.

Over the centuries, problems in Celestial Mechanics have been fostering the developments of new approaches that end up being applied in other fields. However, technology keeps bringing new contribution to allow the development of new and more effective space observation tools. As a result, new phenomena keep being identified, that require a re-reading of current theories and the development of new approaches to proper explain their dynamics.

Here, in this special issue, we put together 15 contributions that focus on those phenomena, so that they are still a challenge to be understood properly even using the up to date approaches. These challenges foster the development of new problem solving methodologies that could also originate from other areas in multidisciplinary context. It is expected that the EJP-ST readers could be encouraged by these challenges and so give their contributions. As so, let us now introduce the papers and the scenario that allow us to classify them as a challenge in Celestial Mechanics.

Geodesy and Geophysics of Mercury: Prospects in view of the BepiColombo Mission, by Hauke Hussmann, Alexander Stark [3], analyses the BepiColombo mission, which is a mission to the planet Mercury. The main studies will start in the spring of 2026. Two orbiters, the Mercury Planetary Orbiter (MPO) and the Mercury Magnetospheric Orbiter (MMO) will be placed in their final orbits in late 2025. There are several particularities to be studied in Mercury, like the large iron core overlaid by a thin silicate mantle and crust, its unique 3:2 spin-orbit coupling and its intrinsic magnetic dipole field. From some libration measurements it is expected that Mercury's outer core is liquid, decoupling the silicate mantle from the deep interior. The paper shows the current knowledge on the evolution of Mercury, with emphasis on its dynamical, rotational and orbital states.

On the rotational motion of NEAs during close encounters with the Earth, by Luiz A.G. Boldrin, Rosana A.N. Araujo, Othon C. Winter [4], studies the rotational motion of the Near-Earth Asteroids (NEAs). The main reason for these studies is that they have numerous close encounters with Earth, Mars and Venus. It has been observed that these close encounters can modify the asteroid's spin state, increasing or decreasing its value, depending on its initial conditions. Those close encounters also make variation in the precession and nutation of the asteroid. It is used a numerical model to compute the variation of the obliquity and the variation of the spin period due to the closer encounters. The results showed significant changes in obliquity and spin period only in the cases with strong encounter. The results also show that the variation of the rotational motion due to the close encounter is capable of tumbling the axis of rotation and gravitational rupture may occur in a few cases.

Some simple results about the Lambert problem, by Alain Albouy and Antonio J. Ureña [5], gives simple proofs of some simple statements concerning the Lambert problem The first results in the known existence and uniqueness results for the Keplerian arc. It is also proved that, in some cases, the time elapsed is a convex function of natural parameters. Their statements and proofs are valid for the three types of Keplerian conic section, orbits: elliptic, parabolic and hyperbolic. The non-uniqueness results and non-convexity results are also proved. The paper does not develop any algorithm of solution, but is limited to some simple, but useful, a priori questions: How many solutions should we expect? Can we be sure that the Newton method will converge? 
Reducing the risk of space debris collision using conditions or performance simultaneous operation in minimum time, by Antonio Delson C. de Jesus and Gabriel Luiz F. Santos [6], studies maneuvers to reduce the risk of collision of space vehicle missions with space debris. The possibilities include sending of tug vehicles to capture debris and sending them to the atmosphere to be destroyed. Rendezvous maneuvers are studied in that scope. It includes the derivation of a tensor equation to solve this problem.

An Optimization Approach to Search for Quasi-Critical Inclinations for Direct and Retrograde Orbits, by Maria Lívia Galhego Thibes Xavier Costa, Rodolpho Vilhena de Moraes, Antônio Fernando Bertachini de Almeida Prado and Jean Paulo dos Santos Carvalho [7], studies the problem of orbits for a spacecraft near the critical inclination for both direct and retrograde orbits. As an addition to the most common approaches, sectoral terms are included in the disturbing function, so the equations of motion become coupled. In this situation the search for critical inclinations is not very easy. The paper then proposes the use of quasi-critical inclinations. In this way, quasi-critical inclinations orbits for direct and retrograde cases are found through using the Hamiltonian formalism and the tools of the nonlinear optimization for artificial satellites around. Io is used as an example.

Tidal friction in satellites and planets. The new version of the creep tide theory, by Sylvio Ferraz-Mello, Cristian Beaugé, Hugo A. Folonier, Gabriel O. Gomes [8], focuses on the creep tide theory and its applications to satellites and planets. In particular, it gives a new set of differential equations to allow easier numerical studies. The creep tide theory models the deformations of a body as a low-Reynolds-number flow instead of defining a fixed form. It is used as an approximate solution of the Navier-Stokes equation, which is dependent on the viscosity of the body. This theory can explain the tidal dissipations of Enceladus and Mimas.

Dynamics of tethered asteroid systems to support planetary defense, by Flaviane C.F. Venditti, Luis O. Marchi, Arun K. Misra, Diogo M. Sanchez, Antonio F.B.A. Prado [9], considers the problem of avoiding an impact of an asteroid with the Earth. It shows a tether assisted methodology to deflect an asteroid by connecting a smaller asteroid to the main one, so altering the center of mass of the system and so moving the larger asteroid to a different orbit where it does not collide with the Earth. A positive point of this technique is that it does not result in fragmentation. The dynamics of the system is analyzed, and the modification of the orbit is measured for several initial conditions. The results show that the method can be used for planetary defense.

An unaccounted component on the Prometheus and Pandora offset angular positions, by Thamiris de Santana, Othon C. Winter and Daniela C. Mourão [10], studies the fact that Prometheus and Pandora are not in their expected angular positions. The usual explanation is the chaotic motion associated with 121:118 mean motion resonance between the satellites. This paper shows that it is necessary to find other mechanisms beyond the mutual interaction between the satellites to explain the observations available at the present time. It is shown that it is necessary to explain an additional variation of mean motion of about $0.45^{\circ} /$ year in one of the satellites for about 20 years.

A semi-analytical approach using the single and double averaged methods and the Lidov-Kozai mechanism, by Jean Paulo dos Santos Carvalho, Rodolpho Vilhena de Moraes [11], analyse the orbital motion of artificial satellites that are orbiting Mercury using a mathematical model that includes the non-sphericity (J2, J3, C22) of Mercury and the third body perturbation. A comparison of the results using single and double averaged models is made. It is shown that, for Mercury, the two models are in agreement, but the single-averaged model is more realistic because it keeps more terms in the disturbing potential. 
Attitude determination by unscented Kalman filter and solar panels as sun sensor, by Leandro Baroni [12], proposes a new method to perform attitude determination and control of CubeSats. The idea is based in the use of photovoltaic panels to replace sun sensor. The paper presents an attitude estimation method based on an unscented Kalman filter, using low cost gyroscope, magnetometer sensors and solar panels to act as a sun sensor. Simulations are made considering the effects of the albedo of the Earth on solar panels, and the results obtained are compared to the ones obtained using an extended Kalman filter.

Dynamical trapping in the area-preserving Hénon map, by Vitor Martins de Oliveira, David Ciro and Iberê Luiz Caldas [13], studies the stickiness, which is a phenomenon in which chaotic orbits expend a long time in some specific regions of the chaotic sea. It is proposed that the different scenarios can be explained by analyzing the crossings between invariant manifolds. It is used as an adaptive refinement procedure to obtain approximated sets of homoclinic and heteroclinic intersections for the area-preserving Hénon map for a generic parameter interval. The results show that these sets have very different statistical properties when the system is highly influenced by dynamical trapping, and they present similar properties when stickiness is almost absent.

Effects of the rotation of a spacecraft in an atmospheric close approach with the Earth, by Jhonathan O. Murcia Piñeros, Vivian M. Gomes, Walter Abrahão dos Santos, Justyna Golebiewska [14], studied the effects of the rotation of a spacecraft when making an "Aero-Gravity Assisted Maneuver". The rotation generates a variable ballistic coefficient, which interferes in the results of the maneuver. To make a more complete study, several values for the masses, sizes and angular velocities are used. The dynamics includes the atmosphere of the Earth, the gravitational fields of the Earth and the Sun and the rotating spacecraft. The main goal is to calculate the variations of energy of the spacecraft as a function of the angular velocity of the spacecraft.

Effects of immersed moonlets in the ring arcs of Saturn, by Gustavo Madeira, Silvia Maria Giuliatti Winter [15], studied the rings of Saturn and they association with some small satellites immersed on them. This study is made by computing the velocity of the mass production and the perturbation caused by the moonlets in the particles of the arc. As an example of the results, it is shown that Aegaeon, Anthe and Methone did not act as sources for their arcs. Some hypothetical scenarios are also analyzed to see under which conditions the moonlets are important in the formation of the rings.

Effects of Higher-Order Multipoles of the Lunar Disturbing Potential on Elongated Orbits in Cislunar Space, by Aaron Jay Rosengren, Hossein Namazyfard, Giorgio E.O. Giacaglia [16], studies orbits in the cislunar space. The effects of the third or higher-order harmonics in the solar disturbing function are considered in the mathematical model. Then, the effects of these terms on highly elliptical orbits, in the situations where the semimajor axis is large are investigated. In particular, orbits near the regions of cislunar space, where the octupole-order approximation is a valid approximation of the gravitational interactions are considered.

Mid-Range Natural Orbits Around the Triple Asteroid 2001 SN263, by Marina Pires de Oliveira Cavalca, Vivian Martins Gomes, Diogo Merguizo Sanchez [17], searches for natural trajectories to place a spacecraft which is the goal of the mission and collect data from the triple asteroid 2001S N263. In particular, it searches for mid-range distance orbits, which are very useful for first observations. The triple asteroid is modelled as a single ellipsoid body, mass of which is assumed to be the sum of the masses of the three bodies. The dimension is assumed to be the size of the orbits of Beta and Alpha. The mathematical model is based in the two-dimensional 
elliptic restricted three-body problem with the addition of the perturbations of the irregular gravity fields (J2) and the solar radiation pressure.

The authors wish to express their appreciation for the support provided by grants \#406841/ 2016-0, 432513/2018-3 and 301338/2016-7 from the National Council for Scientific and Technological Development (CNPq); grants \#2016/24561-0 from São Paulo Research Foundation (FAPESP) and the financial support from the Coordination for the Improvement of Higher Education Personnel (CAPES).

\section{References}

1. V.G. Szebehely, H. Mark, Adventures in Celestial Mechanics, 2nd edition (John Wiley \& Sons, Inc, 1998)

2. C.F. de Melo, et al., J. Phys.: Conf. Ser. 1365, 011001 (2019)

3. H. Hussmann, A. Stark, Eur. Phys. J. Special Topics 229, 1379 (2020)

4. L.A.G. Boldrin, R.A.N. Araujo, O.C. Winter, Eur. Phys. J. Special Topics 229, 1391 (2020)

5. A. Albouy, A.J. Ureña, Eur. Phys. J. Special Topics 229, 1405 (2020)

6. A.D.C. de Jesus, G.L.F. Santos, Eur. Phys. J. Special Topics 229, 1419 (2020)

7. M.L.G.T.X. Costa, R.V. de Moraes, A.F.B. de Almeida Prado, J.P. dos Santos Carvalho, Eur. Phys. J. Special Topics 229, 1429 (2020)

8. S. Ferraz-Mello, C. Beaugé, H.A. Folonier, G.O. Gomes, Tidal friction in satellites and planets. Eur. Phys. J. Special Topics 229, 1441 (2020)

9. F.C.F. Venditti, L.O. Marchi, A.K. Misra, D.M. Sanchez, A.F.B.A. Prado, Eur. Phys. J. Special Topics 229, 1463 (2020)

10. T. de Santana, O.C. Winter, D.C. Mourão, Eur. Phys. J. Special Topics 229, 1479 (2020)

11. J.P. dos Santos Carvalho, R.V. de Moraes, Eur. Phys. J. Special Topics 229, 1491 (2020)

12. L. Baroni, Eur. Phys. J. Special Topics 229, 1501 (2020)

13. V.M. de Oliveira, D. Ciro, I.L. Caldas, Eur. Phys. J. Special Topics 229, 1507 (2020)

14. J.O.M. Piñeros, V.M. Gomes, W.A. dos Santos, J. Golebiewska, Eur. Phys. J. Special Topics 229, 1517 (2020)

15. G. Madeira, S.M.G. Winter, Eur. Phys. J. Special Topics 229, 1527 (2020)

16. A.J. Rosengren, H. Namazyfard, G.E.O. Giacaglia, Eur. Phys. J. Special Topics 229, $1545(2020)$

17. M.P. de Oliveira Cavalca, V.M. Gomes, D.M. Sanchez, Eur. Phys. J. Special Topics 229, $1557(2020)$ 